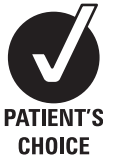

CHOICE

\title{
Abusive behaviour experienced by family carers from people with dementia: the CARD (caring for relatives with dementia) study
}

\author{
C Cooper, A Selwood, M Blanchard, G Livingston
}

Department of Mental Health Sciences, UCL, Charles Bell House, London, UK

\section{Correspondence to}

Dr Claudia Cooper, Department of Mental Health Sciences, UCL, 67-73 Riding House Street, 2nd Floor, Charles Bell House, London W1W 7EJ, UK

Received 4 August 2009 Revised 27 September 2009 Accepted 28 September 2009

\begin{abstract}
Background The authors report the first study of abusive behaviour by people with dementia towards their family carers. The authors hypothesised that while abusive behaviour would be associated with the carer reporting a less rewarding relationship, this could be mediated by the carer's coping style.

Methods The authors interviewed 220 consecutively referred family dementia carers from five UK Community Mental Health Teams, using the revised Modified Conflict Tactics Scale to measure abuse, and the Relationship Rewards Scale.
\end{abstract}

Results 82 (37.3\%) carers reported abuse from the care recipient 'at least sometimes' over the last 3 months. 80 (36.4\%) reported psychologically, and 13 (5.9\%) physically abusive behaviour. On average, current carer relationship rewards had decreased from premorbid levels (mean difference -1.5 (95\% $\mathrm{Cl} 1.8$ to 1.2); $p<0.001)$. The association between higher abuse score and lower current relationship rewards was mediated by dysfunctional coping use. In our final model, current relationship rewards were predicted by reporting a better past relationship ( $\beta=0.66$ (95\% Cl 0.55 to 0.77$)$ ), less abuse from the care recipient $(\beta=-0.39(-0.65$ to $-0.13))$ and fewer dysfunctional coping strategies $(\beta=-0.98(-1.50$ to -0.46$))$.

Conclusion Over a third of family carers reported significant abuse from the people they cared for. Carers who reported more abuse also reported a greater deterioration in their relationship with the person with dementia. The extent to which carers used dysfunctional coping strategies partially explained this, suggesting that interventions to change the carers' coping styles might alleviate the impact of abusive behaviour.

\section{INTRODUCTION}

Carers for people with dementia experience more stress, anxiety and depression than the general population ${ }^{12}$ and carers for people with other disorders. ${ }^{3}$ The particularly distressing nature of dementia caring is unsurprising, given the inevitability of increasing dependence, and high frequency of personality changes, loss of insight and behavioural problems including aggression. A large USA study reported aggression or agitation in $23.7 \%$ of people with dementia in the USA, using the aggression subscale of the Neuropsychiatric Inventory. ${ }^{4}$ Similarly, $20 \%$ of people with Alzheimer's disease in UK studies were reported to be behaving aggressively. 56

We have frequently found in our clinical work that carers report experiencing abuse from the person they care for, and that this is particularly distressing, although the prevalence of such behaviours is unknown. Family carers for younger adults with mental illness have reported high levels of abuse from the people they care for. In a UK Community Mental Health Team (CMHT) survey, $22 \%$ of family carers reported that they had been threatened with violence, and $42 \%$ verbal abuse, by the care recipient (CR) in the last year. ${ }^{7}$ Higher rates of abuse were associated with poor relationships between patients and their families, and a patient history of poly-drug misuse and previous criminal offences. Relatives experiencing higher levels of abuse reported more distress and carer burden.

This study is the first to investigate the prevalence of abuse experienced by family carers of people with dementia. We use the expression 'abusive behaviour' throughout. We are not, however, suggesting by this term that the person with dementia intended to abuse. We determined whether abuse experienced by family carers of people with dementia was related to how rewarding they found their relationship with the CR. Some abusive behaviour may be a symptom of neuropsychiatric symptoms which are part of the dementia, or family stress that can be alleviated by medical or psychosocial interventions. In other cases, it may be more intractable and related to the relationship between the carer and CR, or coping strategies used to manage difficulties which predate the dementia. We know, for example, that emotion-based coping strategies can mitigate the impact of burden on dementia carers' mental health. ${ }^{8}$ In this study, we tested our hypotheses that abusive behaviour would be associated with lower current relationship rewards, after controlling for premorbid relationship rewards, and that the strength of this association would be mediated by carer use of dysfunctional coping strategies.

\section{METHODS}

This study is part of a larger study, and we have reported more details in earlier papers. ${ }^{9-11}$

\section{Setting and participants}

We recruited consecutively referred people with a clinical diagnosis of dementia; living at home and referred to Community Mental Health Teams covering London and Essex (UK). They included inner city, suburban and rural areas. We included those who had an identified family carer, who was providing care for $\geq 4 \mathrm{~h} /$ week. Interviews took 
place at a time and place convenient to the carer, usually their home.

\section{Data collection}

The study received Research Ethics Committee approval. Participants gave written informed consent. The information sheet specified that 'we respect confidentiality but cannot keep it a secret if anyone is being seriously harmed.' CR were asked for consent to access their medical notes, but not interviewed. Where the CR lacked capacity to consent (judged from their psychiatrist and carer's reports), we asked carers whether they thought that CR would have agreed when they had capacity. Three experienced psychiatrists carried out interviews between January 2007 and April 2008

\section{Measures}

We collected sociodemographic data on: carer and CR age, sex, ethnicity, relationship to the CR (spouse, child, other); hours caring/week over preceding few weeks (recorded as 168 if the coresident carer reported caring all the time and could not leave).

Our main effect measure was the carer completed Modified Conflict Tactics Scale (MCTS), ${ }^{12}{ }^{13}$ asking how often in the last 3 months each of five psychologically and five physically abusive behaviours had occurred in the relationship, on a Likert scale from 0 to 4 (never-all the time). A score of $\geq 2$ (sometimes) on one question denotes significant abuse. Carers were asked these questions regarding their behaviour towards the CR. For this part of the study, we also asked carers whether the same behaviours were directed towards them by the CR. Psychometric properties have been reported for the original version. ${ }^{12} 13$ We report psychometric properties of the way we used this scale below.

Our main outcome measure was the Relationship Rewards Scale. This measures the carer's rating of how rewarding their relationship with the CR was before the onset of memory problems, using a four-item measure of relationship rewards. ${ }^{14}$ Carers completed the Brief COPE's (Coping Orientations to Problems Experienced $)^{15} 16$ three subscales: problem-focussed, emotion-focussed and dysfunctional coping.

Carers also completed Bristol activities of daily living (ADL) inventory ${ }^{17}$ and the Neuropsychiatric Inventory (NPI). ${ }^{18} \mathrm{We}$ asked how many alcohol units the CR drank in an average week. We recorded the CR's service receipt using the Client Service Receipt Inventory, ${ }^{19}$ and calculated hours of in home and out of home social care. We obtained the most recent MMSE score ${ }^{20}$ from the CR's medical notes.

\section{Data analysis}

We used SPSS (SPSS, Chicago, Illinois) version 14.0 to analyse data, two-tailed tests and a significance level of $1 \%$ throughout. We defined abuse using the MCTS criteria of a score of $\geq 2$ for caseness (see Methods). We measured the concurrent validity of CR abuse score by examining the degree of correlation with NPI aggression score, and examined the internal consistency of the scale. We compared recent and past relationship reward scores using a paired t test.

For univariate analyses, we used Spearman $\rho$ correlations and Mann-Whitney $U$ tests for non-parametric data, and Pearson correlations and independent $t$ tests for parametric data, which we defined as skewness statistic $<1$ and $>-1$. We entered the sociodemographic, carer and CR characteristics that approached significance $(p<0.1)$ on univariate analyses in their association with either the effect (CR abuse) or outcome measure (recent relationship rewards score) in a forwards linear regression with relationship rewards as the dependent variable. Because we included past relationship rewards in the regression, our outcome measure of current relationship rewards could in fact be interpreted as a measure of change in relationship rewards between the time before the person with dementia had memory problems and the last few months. We assessed tolerance for each predictor and considered that values $<0.10$ may indicate collinearity. $^{21}$ We used generally accepted criteria ${ }^{22}$ to define mediation. These would require that abuse score was correlated with recent relationship rewards score and with our putative mediator, dysfunctional coping; and second that standardised $\beta$ for the relationship of abuse score to relationship rewards score is reduced by adding the mediator to a model with relationship rewards score as the dependent variable. If the relationship between abuse and relationship rewards was no longer significant after adding the mediator, full mediation is said to have occurred; if it is reduced but still significant, this is partial mediation.

\section{RESULTS}

Two hundred and twenty out of three hundred and nineteen (69\%) eligible carers participated, and 98 refused or were uncontactable. There were no differences between participants and non-participants in CR or carer gender $\left(\chi^{2}=1.3, p=0.26\right.$; $\left.\chi^{2}=0.0, p=1.00\right)$; whether they lived together $\left(\chi^{2}=2.2, p=0.14\right)$, or the relationship (partner, child or other) between them $\left(\chi^{2}=2.7, p=0.26\right)$.

\section{Characteristics of the sample}

One hundred and forty-four (65.5\%) family carers were female, $182(82.7 \%)$ were of white UK ethnicity, and 118 (53.6\%) were living with the person with dementia for whom they provided care. Their mean age was 61.7 (SD 13.1; range 24-92 years). One hundred and twenty (54.5\%) were caring for a parent, 72 $(32.7 \%)$ for a spouse and $28(12.7 \%)$ for another relative or friend. One hundred and fifty-nine $(72.3 \%)$ of the CRs were female, and their mean age was 81.6 (SD 7.8; range 58-99). Of the 215 CR for whom a diagnosis was available, 131 (60.9\%) developed Alzheimer's disease $(\mathrm{AD})$ or mixed $\mathrm{AD} /$ vascular dementia; 48 (22.3\%) vascular dementia; nine (4.2\%) Lewy Body or Parkinson's disease dementia; and 27 (12.6\%) other types of dementia. The mean MMSE score was 18.4 (SD 6.8; range 0-29). The median number of units of alcohol consumed by the CR per week was 0 (range $0-84$ ).

\section{Psychometric properties of the abuse scale}

The Cronbach $\alpha$ for the abuse scale was 0.75 . Abuse score correlated with NPI aggression score $(r=0.61, p<0.001)$, demonstrating concurrent validity.

\section{Prevalence}

Nearly half $(47.3 \%: n=104)$ of carers reported that the person they cared for was abusive at any time in the last few months, and $82(37.3 \%)$ of carers reported that the CR was acting abusively 'at least sometimes.' Eighty (36.4\%) reported a psychologically abusive behaviour, and 13 (5.9\%) a physically abusive behaviour (see figure 1 for details of the behaviours included and their individual frequencies). Those who experienced more abuse were also more likely to report acting abusively towards the CR $(r=0.5(p<0.001))$.

\section{Correlates of abuse}

On univariate analysis, significantly higher abuse scores were reported by those caring for $\mathrm{CR}$ who were male, of non-White 


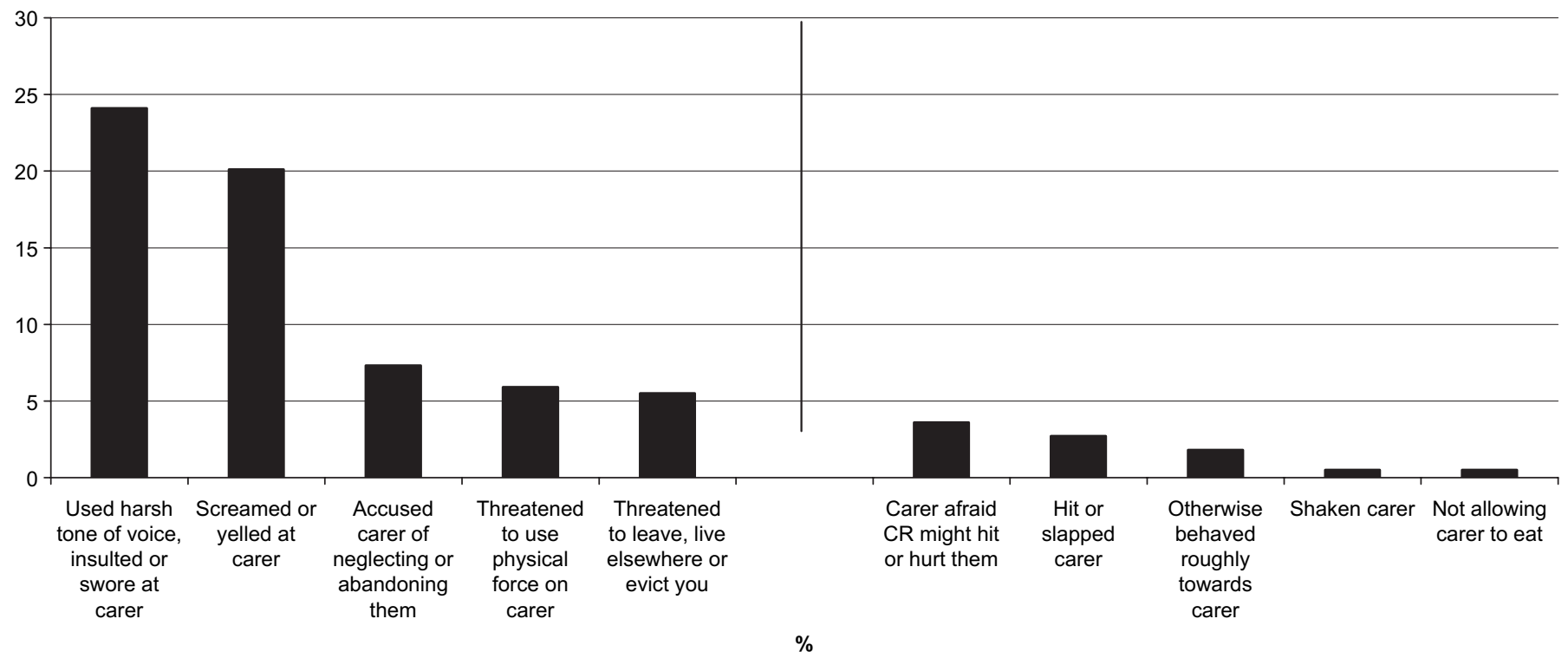

Figure 1 Proportion of family carers who reported that each abusive behaviour was happening 'at least sometimes' in 3 months.

ethnicity and reported to have more ADL impairments. The carers who reported experiencing more abuse used more dysfunctional coping strategies, and reported finding their current and past relationship with the CR less rewarding. Carers who reported more current relationship rewards were caring for $\mathrm{CR}$ with fewer $\mathrm{ADL}$ impairments, received less in-home social care, used fewer dysfunctional coping strategies and found the past relationship more rewarding (see tables 1,2). The current relationship rewards were lower than the premorbid relationship rewards (mean difference -1.5 (95\% CI -1.8 to 1.2 ); $\mathrm{p}<0.001$ ).

In our regression (table 3 ), higher current relationship rewards were predicted by reporting higher past relationship rewards and less abuse from the CR. When we added dysfunctional coping strategy use to the equation, less use of such strategies also predicted higher current relationship rewards, and there was a reduction in the regression coefficient for abuse score, indicating that dysfunctional coping strategies fulfilled criteria as a partial mediator of this relationship (see Data analysis). Tolerance was $>0.80$ for all variables entered.

\section{DISCUSSION}

Aggression is a common symptom in dementia, but this is the first study to investigate prevalence of abuse towards family carers; this may include verbal and psychological, as well as physical abuse. Over a third of family carers we interviewed reported that the person they cared for with dementia was acting abusively towards them at least some of the time. Nearly half of carers reported experiencing any abusive behaviour over the last few months. We have previously reported high rates of abusive behaviour perpetrated by family carers and suggested that health professionals should be asking them routinely about abusive behaviour. ${ }^{9}$ The current study indicates that they should also be asking about abuse the carer may be experiencing themselves from the CR.

Table 1 Association of sociodemographic and other characteristics studied with abuse and recent relationship reward scores

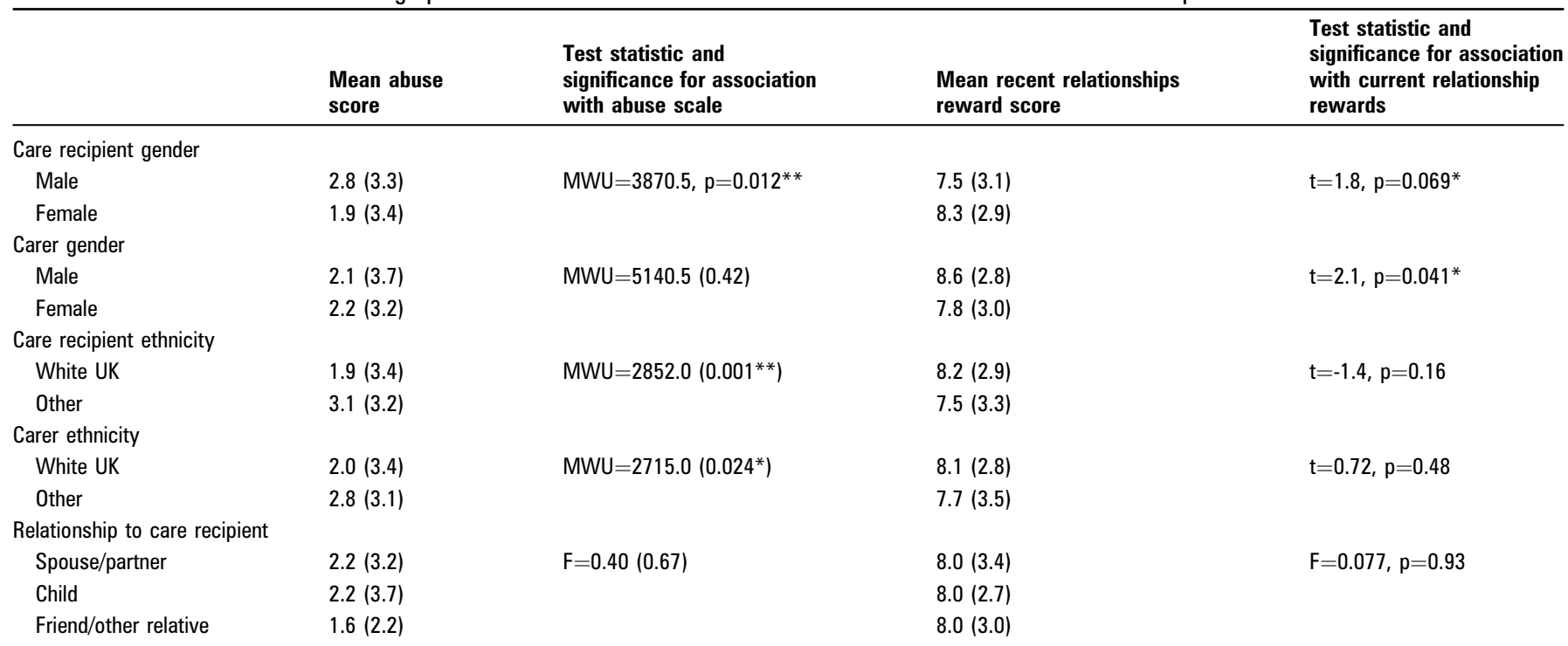

F, ANOVA test statistic; MWU, Mann-Whitney U.

${ }^{*} p<0.1$ (approached significance and included in regression analysis); ${ }^{* *} p<0.01$. 
Table 2 Correlations of sociodemographic and other characteristics studied with abuse and recent relationship reward scores

\begin{tabular}{lll}
\hline & $\begin{array}{l}\text { Correlation coefficient and significance } \\
\text { for association with abuse scale }\end{array}$ & $\begin{array}{l}\text { Correlation coefficient and significance } \\
\text { for association with current } \\
\text { relationship rewards }\end{array}$ \\
\hline Care recipient age & $r=-0.12, p=0.068^{*}$ & $r=-0.021, p=0.76$ \\
Carer age & $r=-0.059, p=0.39$ & $r=0.13, p=0.056^{*}$ \\
Hours caring & $r=0.11,0.094^{*}$ & $r=-0.001, p=0.99$ \\
Mini-Mental State Examination & $r=-0.075, p=0.27$ & $r=-0.044, p=0.52$ \\
Activities of daily living score & $r=0.29, p<0.001^{* *}$ & $r=-0.18, p=0.009^{* *}$ \\
No alcohol units per week & $r=-0.52, p=0.44$ & $r=-0.04, p=0.56$ \\
In home social care & $r=0.072, p=0.29$ & $r=-0.24, p<0.001^{* *}$ \\
Out of home social care & $r=0.052, p=0.44$ & $r=-0.008, p=0.90$ \\
Dysfunctional coping & $r=0.27, p<0.001^{* *}$ & $r=-0.34, p<0.001^{* *}$ \\
Problem-focused coping & $r=0.099, p=0.15$ & $r=-0.074, p=0.28$ \\
Emotion-focused coping & $r=0.097, p=0.15$ & $r=-0.098, p=0.15$ \\
Rating of premorbid relationship & $r=-0.21, p=0.002^{* *}$ & $r=0.67, p<0.001$ \\
Rating of current relationship & $r=-0.32, p<0.001^{* *}$ & - \\
\hline
\end{tabular}

${ }^{*} \mathrm{p}<0.1$ (approached significance and included in regression analysis) ${ }^{* *} \mathrm{p}<0.01$.

We found, as hypothesised, that CR abusive behaviour was associated with the carer reporting fewer current rewards from their dyadic relationship, after controlling for potential confounders and premorbid relationship rewards. We found that while the quality of the premorbid relationship was important, the onset of memory problems had caused a decrease in relationship rewards, and the experience of abuse from the CR was an important predictor of this. Previous authors have debated the extent to which abuse within older couple's relationships represents 'spouse abuse grown old' rather than a new problem. ${ }^{23}$ Our results suggest that both are important, and that where the relationship is strained, interventions to try to reduce abuse may help.

The extent to which carers used dysfunctional coping strategies partially explained the association between abuse and relationship quality. Not all CR abuse can be alleviated through treatment of neuropsychiatric symptoms or provision of more in-home support, and many carers want to continue caring despite verbal and psychological abuse. Our findings suggest that interventions to change the coping strategies carers use may help them to maintain their experience of the relationship as rewarding. This is plausible, as carers who understand neuropsychiatric and abusive symptoms to be part of a dementing illness, rather than attributing them to rejection or ungratefulness, may be more able to continue drawing rewards from their relationship despite the difficulties faced. We have previously found that carers' attributions of symptoms of dementia vary considerably, even when they know the diagnosis. ${ }^{24}$ A manualised intervention designed to promote family carers' use of emotion-focussed and problem-focussed coping strategies and reduce their use of dysfunctional coping strategies was efficacious in reducing carer depression and anger intensity in a USA trial, ${ }^{25-27}$ and may also be effective in maintaining relationship rewards in those experiencing abusive behaviour. CR ethnicity was associated with abusive behaviour on univariate analysis, but this association was no longer significant after controlling for CR morbidity characteristics. This probably reflects the association between non-White ethnicity and later referral (and therefore more neuropsychiatric symptoms at time of referral) to dementia services. ${ }^{28}$

\section{LIMITATIONS}

This was a cross-sectional study, so we cannot confirm directions of causality, and while we hypothesise that dysfunctional coping mediated the relationship between CR abuse and relationship rewards, it could also be a confounder. This was a representative sample of people with dementia newly referred to services, so our sampling frame disproportionately selected those with a new diagnosis or acute problems. Thus, the population was less cognitively impaired than the average population with dementia, ${ }^{29}$ but more patients may have had untreated neuropsychiatric symptoms (although the mean score was almost identical to that of a previous representative sample). ${ }^{6}$ There may have been a social desirability bias, if carers were reluctant to report abusive behaviour they experienced, or that they no longer experienced the relationship with the CR as rewarding. We used the term CR abusive behaviour throughout, but in many cases the CR would not have known that their behaviour may cause distress, so the term does not imply intent. Similarly, many of the carers did not view behaviour that they

Table 3 Significant predictors in forwards linear regression with recent relationship rewards score as the dependent variable

\begin{tabular}{lllr}
\hline & Predictors & Standardised $\beta(+95 \%$ Cl) & p Value \\
\hline Step 2 $\left(\mathrm{R}^{2}=0.48\right)^{*}$ & Past relationship rewards & $0.69(0.58$ to 0.80$)$ & $<0.001$ \\
& Abuse score & $-0.51(-0.77$ to 0.25$)$ & $<0.001$ \\
Step 3 $\left(\mathrm{R}^{2}=0.51\right) \dagger$ & Past relationship rewards & $0.66(0.55$ to 0.77$)$ & $<0.001$ \\
& Abuse score & $-0.39(-0.65$ to 0.13$)$ & 0.003 \\
& Dysfunctional coping score & $-0.98(-1.50$ to 0.46$)$ & $<0.001$ \\
\hline
\end{tabular}

Step 1 is not shown.

*Care recipient and carer gender, ethnicity and age; number of hours caring, activities of daily living score, amount of in-home social care and past relationship rewards were entered in step 1.

†Carer dysfunctional coping scale score was entered in step 2. 
reported on the MCTS scale as abusive. We did not find a significant association between alcohol intake by the CR and abusive behaviour. While the association between alcohol and aggression is well known, this probably reflects the very low levels of alcohol use in this population.

\section{CONCLUSIONS}

Over a third of family carers reported significant abuse from the people they cared for. Carers who reported a greater deterioration in their relationship with the person with dementia also reported more abuse. The extent to which carers used dysfunctional coping strategies partially explained this, suggesting that interventions to change the carers' coping styles might alleviate the impact of abusive behaviour.

Acknowledgements We would like to thank the carers and patients who took part in the study. We are grateful to Camden and Islington NHS Foundation Trust, North Essex Partnership NHS Foundation Trust and North East London NHS Foundation Trust; in particular to RA, SJ, VK and TS. We would like to thank CO, for conducting some of the interviews, and the Alzheimer's Society (Islington Branch) and SN for assistance in developing the study.

Funding This research was funded by a research training fellowship awarded to CC by the Medical Research Council. The author's work was carried out independently of the funders.

Competing interests None.

Patient consent Obtained.

Ethics approval Ethics approval was provided by the London MREC.

Contributors $\mathrm{CC}, \mathrm{GL}$ and $\mathrm{MB}$ conceived and designed the study; $\mathrm{CC}$ analysed the data; all authors (CC, GL, MB and AS) were involved in interpretation of data; CC drafted the article and all authors (CC, GL, MB and AS) revised it critically for important intellectual content and gave final approval of the version to be published. CC will act as guarantor.

Provenance and peer review Not commissioned; externally peer reviewed.

\section{REFERENCES}

1. Pinquart M, Sorensen S. Differences between caregivers and noncaregivers in psychological health and physical health: a meta-analysis. Psychol Aging 2003:18:250-67.

2. Cooper C, Balamurali TB, Livingston G. A systematic review of the prevalence and covariates of anxiety in caregivers of people with dementia. Int Psychogeriatr 2007:19:175-95.

3. Livingston G, Manela M, Katona C. Depression and other psychiatric morbidity in carers of elderly people living at home. BMJ 1996;312:153-6.

4. Lyketsos CG, Steinberg M, Tschanz JT, et al. Mental and behavioral disturbances in dementia: findings from the Cache County Study on Memory in Aging. Am J Psychiatry 2000;157:708-14.

5. Burns A, Jacoby R, Levy R. Psychiatric phenomena in Alzheimers-disease. 4. Disorders of behavior. Br J Psychiatry 1990:157:86-94.

6. Ryu SH, Katona C, Rive B, et al. Persistence of and changes in neuropsychiatric symptoms in Alzheimer disease over 6 months: the LASER-AD Study. Am J Geriatr Psychiatry 2005;13:976-83.
7. Vaddadi KS, Gilleard $\mathrm{C}$. Fryer $\mathrm{H}$. Abuse of carers by relatives with severe mental illness. Int J Soc Psychiatry 2002;48:149-55.

8. Cooper C, Katona C, Orrell M, et al. Coping strategies, anxiety and depression in caregivers of people with Alzheimer's disease. Int J Geriatr Psychiatry 2008;23:929-36

9. Cooper C, Selwood A, Blanchard M, et al. Abuse of people with dementia by family carers: representative cross sectional survey. BMJ 2009;338:b155.

10. Cooper C, Selwood A, Blanchard M, et al. The determinants of family carer's abusive behaviour to people with dementia: results of the CARD study. J Affect Disord 2010;121:136-42.

11. Selwood A, Cooper C, Owens C, et al. What would help me stop abusing? The family carer's perspective. Int Psychogeriatr 2009:21:309-13.

12. Beach SR, Schulz R, Williamson GM, et al. Risk factors for potentially harmful informal caregiver behavior. J Am Geriatr Soc 2005;53:255-61.

13. Cooper C, Manela M, Katona C, et al. Screening for elder abuse in dementia in the LASER-AD study: prevalence, correlates and validation of instruments. Int J Geriatr Psychiatry 2008:23:283-8.

14. Williamson GM, Shaffer DR. Relationship quality and potentially harmful behaviors by spousal caregivers: how we were then, how we are now. Psychol Aging 2001:16:217-26.

15. Cooper C, Katona C, Livingston G. Validity and reliability of the brief COPE in carers of people with dementia: the LASER-AD study. J Nerv Ment Dis 2008:196:838-43.

16. Carver CS. You want to measure coping but your protocol's too long: consider the brief COPE. Int J Behav Med 1997:4:92-100.

17. Bucks RS, Ashworth DL, Wilcock GK, et al. Assessment of activities of daily living in dementia: development of the Bristol activities of daily living scale. Age Ageing 1996;25:113-20.

18. Cummings $\mathbf{J L}$. The neuropsychiatric inventory: assessing psychopathology in dementia patients. Neurology 1997; 48:10-16.

19. Knapp M. The economic evaluation of mental health care. Aldershot: Arena, 1995

20. Folstein MF, Folstein SE, McHugh PR. Mini-mental state. A practical method for grading the cognitive state of patients for the clinician. J Psychiatr Research 1975:12:189-98

21. UCLA Academic Technology Services. UCLA Academic Technology Services 2009. http://www.ats.ucla.edu/stat/spss/webbooks/reg/chapter2/spssreg2.htm (accessed 20 Feb 2009).

22. Baron RM, Kenny DA. The moderator mediator variable distinction in social psychological-research - conceptual, strategic, and statistical considerations. J Pers Soc Psychol 1986;51:1173-82

23. Harris S. For better or worse: spouse abuse grown old. J Elder Abuse Negl 1996; : : $1-33$

24. Paton J, Johnston K, Katona C, et al. What causes problems in Alzheimer's disease: attributions by caregivers. A qualitative study. Int J Geriatr Psychiatry 2004; 19:527-32.

25. Gallagher-Thompson D, Coon DW, Solano N, et al. Change in indices of distress among Latino and Anglo female caregivers of elderly relatives with dementia: sitespecific results from the REACH national collaborative study. Gerontologist 2003;43:580-91

26. Coon DW, Thompson L, Steffen A, et al. Anger and depression management: psychoeducational skill training interventions for women caregivers of a relative with dementia. Gerontologist 2003;43:678-89.

27. Gallagher-Thompson D, Arean P, Rivera P, et al. A psychoeducational intervention to reduce distress in hispanic family caregivers: results of a pilot study. Clin Gerontol 2001;23:17-32.

28. LoGiudice D. Hassett A, Cook R, et al. Equity of access to a memory clinic in Melbourne? Non-English speaking background attenders are more severely demented and have increased rates of psychiatric disorders. Int J Geriatr Psychiatry 2001;16:327-34

29. Fratiglioni L. Epidemiology. In: Wimo A, Jonsson B, Karlsson G, Winblad B, eds. Health economics of dementia. New York: John Wiley \& Sons, 1998:13-31. 